\title{
A SHORT PROOF OF KOWALSKY'S HEDGEHOG THEOREM
}

\author{
MARY ANNE SWARDSON
}

\begin{abstract}
We give a simple proof of Kowalsky's theorem that every metrizable space of weight $m$ is embeddable in a countable product of hedgehogs with $m$ spines.
\end{abstract}

For an infinite cardinal $\mathfrak{m}$, let $J(\mathfrak{m})$ be the set $\{(0,0)\} \cup((0,1] \times \mathfrak{m})$ endowed with the metric

$$
d((x, \alpha),(y, \beta))=\left\{\begin{array}{l}
|x-y| \text { if } \alpha=\beta \text { or } x y=0, \\
x+y \text { otherwise. }
\end{array}\right.
$$

This space is called the hedgehog with $\mathrm{m}$ spines (see [2, pp. 314-315] for further details), and the theorem of Kowalsky stated below asserts that $(J(m))^{\omega}$ is universal for metrizable spaces of weight $m$. Published proofs ([1], [2, Theorem 4.4.9]) obscure the fact that the embedding is simple and transparent. The proof given here seems natural.

TheOREM (KowALSKY). Let $X$ be a metrizable space of weight $m$. Then $X$ is embeddable in $(J(m))^{\omega}$.

Proof. $X$ has a base $\mathscr{B}=\cup_{n \in N} \mathscr{B}_{n}$ where each $\mathscr{B}_{n}=\left\{B_{n \xi}: \xi<\mathfrak{m}\right\}$ is discrete. Each $B_{n \xi}$ is the cozero-set of a continuous function $f_{n \xi}$ with range the unit interval $[0,1]$.

For all $n \in \mathbf{N}$, define $f_{n}: X \rightarrow J(m)$ by

$$
f_{n}(x)=\left\{\begin{array}{l}
\left(f_{n \xi}(x), \xi\right) \text { if } x \in B_{n \xi}, \\
(0,0) \text { if } x \notin \bigcup \mathscr{B}_{n} .
\end{array}\right.
$$

By the discreteness of $\mathscr{B}_{n}$ and the continuity of $f_{n \xi}$, each $f_{n}$ is well defined and continuous. It is easily seen that the family $\left(f_{n}\right)_{n \in \mathrm{N}}$ separates points and closed sets. Thus the diagonal map $\Delta_{n \in \mathrm{N}} f_{n}$ embeds $X$ into $(J(\mathfrak{m}))^{\omega}$.

\section{REFERENCES}

1. H. J. Kowalsky, Einbettung metrischer Räume, Arch. Math. 8 (1957), 336-339.

2. R. Engelking, General topology, PWN, Warsaw, 1977.

Department of Mathematics, Ohio University, Athens, Oho 45701

Received by the editors December 1, 1978 and, in revised form, December 14, 1978. AMS (MOS) subject classifications (1970). Primary 54E35. 